\title{
E-cigs have lower levels of harmful toxins
}

\author{
Cite as: CMAJ 2017 February 27;189:E331. doi: 10.1503/cmaj.1095396
}

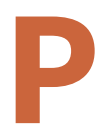

eople who switched from regular cigarettes to e-cigarettes or other nicotine-replacement therapies (NRTs), such as gum or patches, had "substantially reduced levels of measured carcinogens and toxins," found a study published Feb. 7 in the Annals of Internal Medicine.

The study was conducted in the United Kingdom. It followed 181 people for at least six months, and compared the biomarkers of participants who used e-cigarettes or NRTs to those of smokers, as well as to those of people who used e-cigarettes or NRTs while continuing to smoke regular cigarettes. Participants who used only e-cigarettes or NRTs had "significantly lower" levels of tobaccospecific $\mathrm{N}$-nitrosamines and volatile organic compounds, as measured in urine and saliva samples.

This study adds to the the study's findings, telling CBS News that many smokers may have quit in recent years if they weren't misled into thinking vaping was harmful by the "outright incorrect rhetoric towards vapor products" of tobacco-control activists. In the same article, however, Dr. Norman Edelman of the American Lung Association nal of the American Medical Association Pediatrics found that e-cigarette use among young adults and adolescents was "associated with progression to traditional cigarette smoking."

And even if e-cigarettes are safer than smoking, that doesn't mean they are healthy, other researchers have

\section{existing body of evidence}

that suggests e-cigarettes and NRTs are low-risk and "far safer" than smoking, lead author Lion Shahab of University College London told Reuters.

In a press release, the study's primary funder, Cancer Research UK, stated that a better understanding of safer alternatives to tobacco, including e-cigarettes, is an important step in reducing tobaccorelated deaths.

The president of the American Vaping Association, Gregory Conley, welcomed noted that the study only looked at a few toxins and didn't test for any toxins that could be produced by e-cigarettes.

The use of e-cigarettes as a means of smoking cessation and harm-reduction remains a controversial topic. In August 2015, an editorial in The Lancet noted that one highly touted study on e-cigarette safety was "methodologically weak" and had conflicts of interest around funding, leading only to "evidence-based confusion." A November 2015 study in the Jour- noted. A July 2016 study of chemicals in e-cigarette vapor concluded that "harmful emissions are expected to be ubiquitous when e-cigarette vapor is present." In August 2016, at the world's largest cardiology conference, researchers warned that vaping could be as bad for the heart as cigarettes, adding that the long-term effects of e-cigarette use remain unknown.

\section{Roger Collier, CMAJ}

